\title{
Extended sources of the main events of the Umbria-Marche (1997) seismic sequence inverted from geophysical data
}

\author{
Maria Elina Belardinelli \\ Dipartimento di Fisica-Settore di Geofisica, Università degli Studi di Bologna, Italy
}

\begin{abstract}
The three largest events of the 1997 Umbria-Marche (Italy) sequence occurred on September 26, 1997 at 00:33 GMT (Event 1, $\mathrm{M}_{\mathrm{W}}=5.7$ ) and 09:40 GMT (Event 2, $\left.\mathrm{M}_{\mathrm{W}}=6.0\right)$ in the Colfiorito area and on the October 14, 1997 at 15:23 (Event 3, $\mathrm{M}_{\mathrm{W}}=5.6$ ) in the Sellano area. The availability of different sets of geodetic and seismological data allowed several studies to characterize the extended sources of events 1-3. In this work, I review some of the studies that obtain the properties of the seismic sources by inversion of available data. Generally these studies assume the seismic sources as dislocations or distributions of equivalent point sources in elastic half-spaces. Following their chronological order, they model increasing complexities of the sources by using an increasing number of data. Some of the differences between results obtained, such as the top edge depth estimates, are shown to be due to the different approaches used. Commonly a 1-D crustal model is used in inverting strongmotion data. Instead homogeneous elastic half-spaces are mainly assumed in inverting geodetic data to obtain the three main sources of the 1997 Umbria-Marche sequence. Assuming the same crustal structure is important to make comparable results obtained analyzing seismological data or geodetic data separately, as it has been done till now for this sequence.
\end{abstract}

Key words DInSAR - GPS - strong-motion - teleseismic data - seismic source

\section{Introduction}

During the 1997-1998 Umbria-Marche earthquake sequence more than 2000 shocks were recorded, three of which had moment magnitudes larger than 5.7: the September 26, 1997 at 00:33 GMT (Event 1, $\mathrm{M}_{\mathrm{W}}=5.7$ ) and 09:40 GMT (Event 2, $\mathrm{M}_{\mathrm{w}}=6.0$ ) in the Colfiorito area and the October 14, 1997 at 15:23

Mailing address: Prof. Maria Elina Belardinelli, Università di Bologna, Dipartimento di Fisica, Settore di Geofisica, Viale Berti Pichat 8, 40127 Bologna, Italy; e-mail: mariaelina.belardinelli@unibo.it
(Event 3, $\mathrm{M}_{\mathrm{W}}=5.6$ ) in the Sellano area (fig. 1). A reference value of the scalar seismic moment and focal mechanism of the ruptures is given by the moment tensor analysis of long period seismological data from the MedNet network (Ekstrom et al., 1998, CMT solution hereinafter). This analysis suggests normal faulting with a NE-SW tension axis and the presumed fault plane dipping towards SW. Several data sets concerning the coseismic displacement field were available after the three events.

The assessment of the properties of extended seismic sources is a fundamental requirement for further studies aimed at relating the source properties to damage, computing accurate stress transfers and characterizing hazard related to a given seismic province. When this result is obtained through inversion of available data, it is said that a finite-fault inversion is per- 


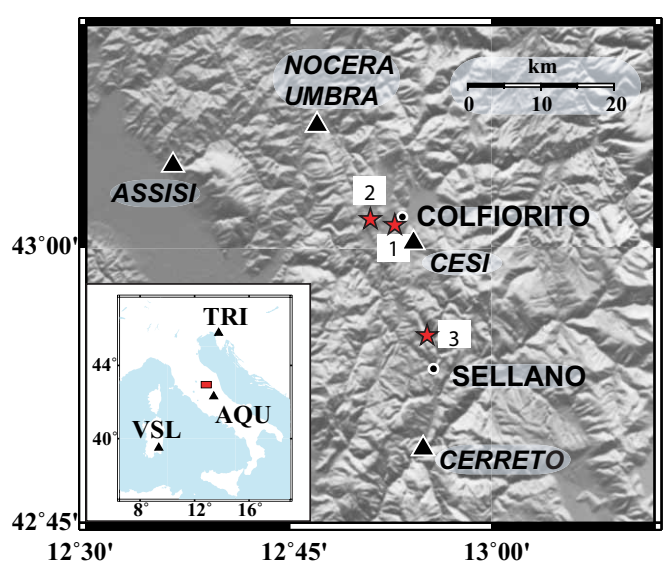

Fig. 1. The epicentres (red stars) of three largest events in the Umbria-Marche (1997-1998) sequence. Strong-motion stations are indicated by black triangles. The inset shows the area in the figure (red rectangle) and the location of broad-band MedNet stations used in UMI studies (black triangles).

formed. We review here the studies that characterize the three seismic sources of events 1-3 by means of inversions of two kinds of data: geodetic data (e.g. Lundgren and Stramondo, 2002; Belardinelli et al.., 2003; De Martini et al., 2003; Hernandez et al., 2004; Santini et al., 2004; Crippa et al., 2006; Dalla Via et al., 2007) and seismological data (e.g. Capuano et al., 2000; Pino and Mazza, 2000; Hernandez et al., 2004). We briefly indicate as UMI studies (Umbria-Marche inversion studies) the finitefault inversions of the three main events of the 1997 Umbria-Marche sequence that will be discussed here.

After an introduction of the data used, this paper will present generalities of the inversion methods and the specific methodological approach followed by studies dealing with geodetic data or seismological data separately. Then the main results achieved on the three main events of the Umbria-Marche sequence will be presented. Some topics about UMI studies versus the state of the art of finite-fault inversions will be discussed in the last section.

It is worth noting that for this sequence no study to date has inverted the source parameters using both seismological and geodetic data jointly, whereas there are several examples of such a kind of inversions for subsequent earthquakes. For instance the $1999 \mathrm{M}_{\mathrm{W}}$ 7.5-7.6 Izmit, Turkey mainshock is studied by Delouis et al. (2002) by inverting GPS, DInSAR, strong-motion and teleseismic data. The 2000 $\mathrm{M}_{\mathrm{W}} 6.6$ western Tottori, Japan earthquake is studied by Piatanesi et al. (2007) by inverting GPS and strong-motion data.

\section{Data selection}

The moderate magnitudes of events 1-3 of the Umbria Marche sequence impose measurements with the largest «signal to noise» ratio within the ensemble of available data. Permanent displacements induced by the three events were measured through the analysis of DInSAR data. During the Colfiorito sequence several interferometric images were acquired by satellites ERS1 and ERS2, but few of them can be organized in pairs of images revealing enough coherence in the area of interest (Lundgren and Stramondo, 2002; Crippa et al., 2006). The difference between the images taken on 1997 September 7 and 1997 October 12 by satellite ERS2 will be called «Colfiorito interferogram» hereinafter. It regards the displacement (in the satellite line of sight or slant range direction) caused by events 1 and 2 and intervening smaller events. Another pair of images taken by ERS2 and ERS1 at 1997 August 9 and 1997 October 17 , respectively, also contains the coseismic displacement caused by event 3 and will be referred to as the "Colfiorito-Sellano interferogram». Finally a pair of images taken by ERS2 on 1997 October 12 and 1997 November 16 basically contains the deformation due to event 3 and will be referred as the "Sellano interferogram» (Lundgren and Stramondo, 2002).

Measures of permanent coseismic displacement as a 3-D vector were obtained at GPS monuments by differentiation of the data collected in two surveys (e.g. Belardinelli et al., 2003). The first survey was taken in 1995 by Istituto Geografico Militare (IGM) and the second survey, between 1997 October 7 and 10, by 
Istituto Nazionale di Geofisica at 13 monuments of the first survey. Of the latter, only four monuments (CAPA, CROC, FOLI and PENN, fig. 2) showed significant horizontal displacement caused by events 1 and 2 and intervening smaller events and only two stations, CROC and COLF, were subjected to a vertical displacement greater in absolute value than the accuracy. Unfortunately, the coseismic displacement errors are affected by the significantly lower accuracy of the first survey compared to the second one. Accordingly, the accuracy of GPS data are estimated as $2.4 \mathrm{~cm}$ and $4.0 \mathrm{~cm}$ for the horizontal and vertical components of displacement, respectively (Belardinelli et. al., 2003). After event 3, no major coseismic deformation at GPS sites was observed (Hernandez et al., 2004). The elevation changes caused mainly by event 2 were also measured along a levelling line surveyed in 1992 and 1998 by IGM (De Martini et al., 2003).

Seismological data consist of measurements of ground acceleration, velocity or displacement as a function of time at the locations of seismic stations. Records of 3-D ground acceleration were provided by strong-motion accelerometers deployed by Servizio Sismico Nazionale (SSN), National Energy Agency (ENEA) and Electric National Board (ENEL). In order to provide the highest level of detail on the rupture process, UMI studies consider the records taken by accelerometers located at distances less than $30 \mathrm{~km}$ from the focal area and having a good azimuthal coverage relative to the fault orientations (taken from the CMT solution). Moreover only the accelerometers providing the simplest waveforms (with the least site-effects) are considered (Capuano et al., 2000) or weighted more than other stations (Hernandez et al., 2004). For events 1 and 2 these accelerometers are located at the stations of Assisi and Cerreto (fig. 1). For event 3, they are located at the stations of Cerreto and Cesi. The latter station, not considered by Hernandez et al. (2004), has been operating only since 1997 October 3. During events 1-3, three broad-band seismic stations of the MedNet regional network, at distances less than $500 \mathrm{~km}$ from the focal area (AQU, TRI and VSL, fig. 1), recorded good quality (high signal-to-noise

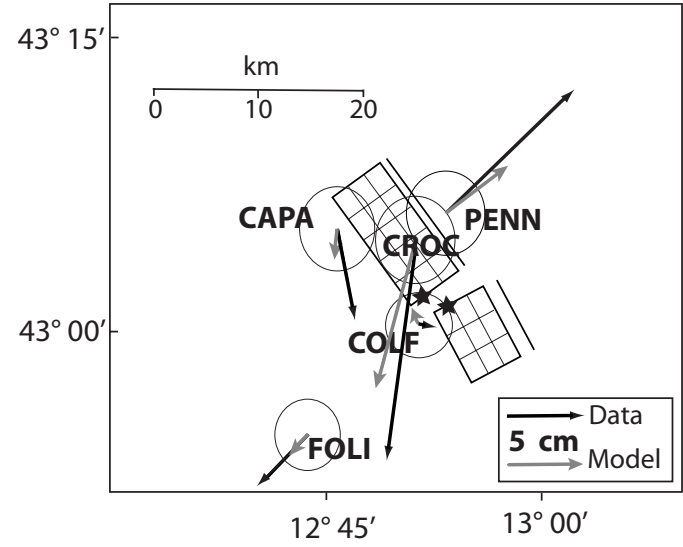

Fig. 2. Geometry assumed by Hernandez et al. (2004) for the sources of events 1 and 2. GPS data of horizontal displacement recorded in near field stations are compared to model results (best model according to both DInSAR data and GPS data). The figure shows only five out of twelve GPS stations used in the inversion (see text). The epicentres of events 1 and 2 are indicated with black stars. The fault discretization used is also shown in map view. The solid lines parallel to fault strikes represent the intersections of the prolongation of the fault planes with the Earth surface. The top edge depth of the fault of event 2 is fixed to $700 \mathrm{~m}$. The crustal model assumed is reported in Table I. Figure modified from Hernandez et al. (2004).

ratio) waveforms of 3-D ground displacement as a function of time (e.g. Pino and Mazza, 2000). Station AQU is also conveniently placed to show directivity effects, having an azimuth close to the strike of the fault planes involved in the seismic ruptures (figs. 1 and 2).

\section{Methodological approaches}

\subsection{Generalities}

We may schematize an inversion problem as it follows. Let $m$ be a vector containing the parameters to be inverted (or free parameters), $c$ the vector of parameters fixed or assumed as known during the inversion. The inversion de- 
termines the parameter $m$ by comparing the available data organized in a vector $d$ with their estimates $d^{\text {mod }}$ provided by a model equation

$$
d^{m o d}=g(m ; c)
$$

where $\mathbf{d}^{\text {mod }}$ is the vector containing the model estimates. The inversion is performed by minimizing a misfit or cost function that, following the approach of Belardinelli et al. (2003), can be expressed as

$$
\varepsilon(m)=\left(d^{m o d}-d\right)^{T} C^{-1}\left(d^{m o d}-d\right)
$$

where $C$ is the covariance matrix whose elements contain data uncertainties. The vector of free parameters retrieved from the inversion, $m_{I}$, also called «inverted model», is such that: $\varepsilon\left(m_{I}\right)=\min \{\varepsilon(m)\}$, with varying $m$ in the parameter space. In order to find the minimum value of the misfit function, the exploration of the parameter space is performed starting from a given set of parameters $m_{s}$, the so-called starting model. If the model equation is linear in $m$, i.e. in (3.1) $g(m ; c)=G(c) m$, where $G$ is a matrix, then the inversion is linear and $m_{I}$ satisfies the so called «least squares» criterion. In this case, the retrieved parameters can be expressed analytically as it follows

$$
\left.m_{I}=\left(G^{T} C^{-1} G\right)^{-1} G^{T} C^{-1} d\right)
$$

When the model equation is nonlinear in $m$ the inversion is said non linear and the misfit function can be characterized by several local minima. In this case, a local exploration of the parameter space, such as a trial and error exploration, may determine estimates of parameter values $m_{I}$ which depend on the starting model. This occurs when $m_{I}$ is incorrectly identified with the point in the parameter space realizing the local minimum of the misfit function that is closest to the starting model, $m_{s}$. To avoid this, it is better to perform a global exploration of the parameter space, by means of genetic algorithms, for instance. The latter algorithm is used by Hernandez et al. (2004) to generate different models of slip distribution of events 1 and 2 that are in agreement with geodetic observations within the data accuracy (i.e. they represent models that are consistent with data). The resulting model population is used to evaluate the resolution of the inverted model along the fault planes, as will be discussed later in section 4 . Also simulated annealing algorithms are suitable to escape local minima of $\varepsilon$ and to find the absolute minima in nonlinear inversions. Inversions using simulated annealing (Belardinelli et al., 2003 for event 2, Lundgren and Stramondo, 2002, for events 1-3) are a particular case of Monte Carlo inversions, where trial sets of parameters $m$ are generated randomly (Santini et al., 2004, for events 1-3).

In order to improve the resolution and the stability of the inversion obtained with a single data set, it is possible to perform a joint inversion of different kinds of data. In a joint inversion, following the notation of Belardinelli et al. (2003), the cost function is obtained by assigning a different weight to different data by means of a weight matrix $W$, as it follows

$$
\varepsilon=\left(d^{m o d}-d\right)^{T} W C^{-1}\left(d^{\text {mod }}-d\right)
$$

The weight matrix is diagonal and it should be chosen in such a way that the cumulative contribution to the cost function of each data set is comparable, regardless of the number of data contained in the different data sets.

\subsection{Parameter selection and data treatment}

Inversions of geodetic data allow the determination of parameters that characterize the fault from a static point of view. These are the fault geometry (location, focal mechanism, maximum length and width) and the final distribution of the slip vector over the fault plane. In addition to the parameters mentioned above, inversions of seismological data determine parameters that characterize the fault from a kinematic point of view, such as the distributions over the fault plane of the rupture time (or the rupture velocity) and the rise time. In the latter case, generally the same source time function over the whole fault is assumed. The larger number of parameters that can be inverted from seismological data is justified because, for each component of ground displacement at every 
seismic station, seismological data are in the form of a time series instead of the single value provided by geodetic data.

We shall see that, given the restricted number of good-quality available data discussed in section 2, in order to limit the number of free parameters, most of UMI studies further restrict the number of free parameters with respect to what is stated above, by making a priori explicit assumptions. For instance, apart from Lundgren and Stramondo (2002) who also invert for the direction of the slip vector in each point of the extended sources (fig. 3), most UMI studies assumed a seismic source with a homogeneous rake angle. In so doing, the slip distribution is meant as the slip amplitude distribution over the fault plane. Geodetic data are used to invert the slip amplitude distributions by Hernandez et al. (2004); Santini et al. (2004), Crippa et al. (2006) and Dalla Via et al. (2007). It is worth recalling that in principle, this kind of inversion is linear, since the model displacement, $d^{\text {mod }}$, is linked to the vector $m$ of slip values on the fault plane, through a linear Green operator, $G$. Apart from Hernandez et al. (2004) who also invert for the rupture time distribution of events 2 and 3 , UMI studies dealing with seismological data assume a homogeneous rupture velocity vector over the fault plane, to be determined from the inversion. All UMI studies dealing with seismological data assume a homogeneous rise time value, eventually vanishing (Pino and Mazza, 2000; Capuano et al., 2000).

Some UMI studies perform a joint inversion of different kinds of geodetic data. The Colfiorito interferogram and GPS data are jointly inverted by Belardinelli et al. (2003), for event 2 and Hernandez et al. (2004), for events 1 and 2. For events 1-3, Lundgren and Stramondo (2002) perform a joint inversion of different DInSAR image pairs and GPS data in order to better resolve horizontal components of the slip vector (fig. 3).

\subsubsection{Inversion of geodetic data}

Unlike seismological data, geodetic data refer to temporal windows encompassing more than one seismic event of the Umbria-Marche se-
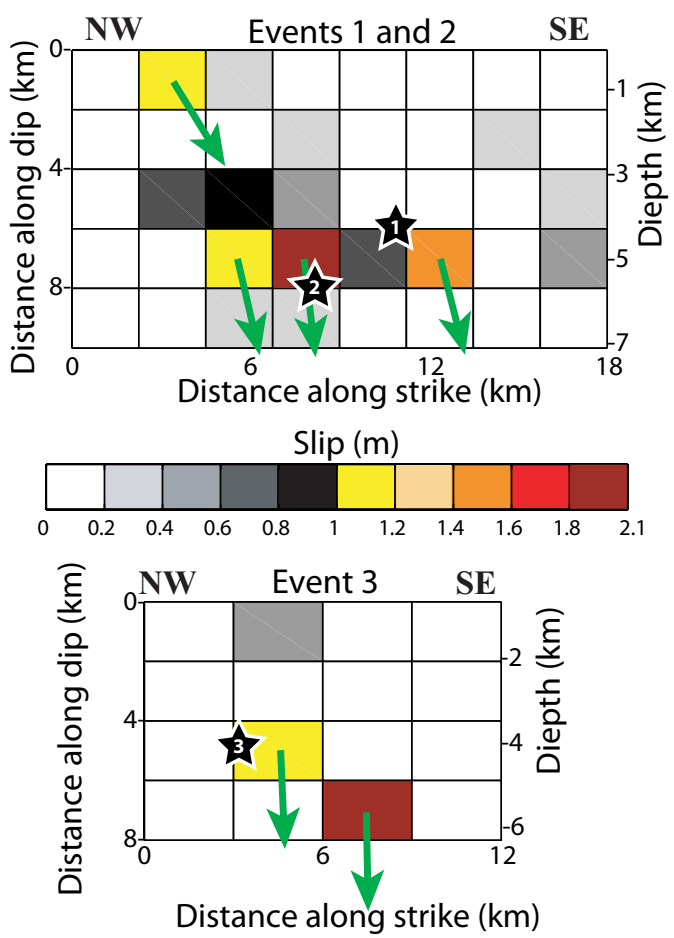

Fig. 3. Joint inversion of GPS data and DInSAR data performed by Lundgren and Stramondo (2002) for the slip distribution of events 1 and 2 (top) and event 3 (bottom). The inverted direction of slip (normalized vectors) is shown in the patches of slip larger than $1 \mathrm{~m}$ (green arrows). Black stars represent vertical projections of the observed epicentres on the inverted fault planes. Events 1 and 2 are assumed on the same fault plane (see section 3.2.1.). Faults are assumed as rectangular dislocations in an elastic homogeneous half-space.

quence (see section 2). In principle these data view a displacement field caused by events 1-3 and the smaller events, intervening between the times of the first and the second GPS survey (or between SAR images). However the contribution of minor events to the observed displacement is much smaller than that of events 1-3. The minor intervening events are either neglected (Lundgren and Stramondo, 2002; Hernandez et al., 2004; Crippa et al., 2006; Dalla Via et al., 2007) or re- 
solved as a part of the inversion for events 1-3 (Santini et al., 2004 under the assumption that minor events roughly belong to the same fault zone of events 1-3) or explicitly taken into account as fixed parameters, $c$, together with event 1 parameters. The latter procedure is followed by UMI studies inverting for event 2 only (Belardinelli et al., 2003; De Martini et al., 2003), that choose $\mathbf{c}$ in agreement with previous studies.

Forward modelling of events 1 and 2 is made by Hunstad et al. (1999) in order to reproduce GPS data, in particular their study focuses on the heterogeneous slip distribution, the top edge depth and the rake of event 2. Details concerning the slip distribution of events 1 and 2 are provided by inversions of the Colfiorito interferogram (Lundgren and Stramondo, 2002; Hernandez et al., 2004; Santini et al., 2004; Crippa et al., 2006; Dalla Via et al., 2007). In these cases, either a fixed geometry of the fault (location, focal mechanism and maximum dimensions) is assumed in agreement with previous studies or some of the fault parameters are chosen as it follows. Trial values of these parameters are put in the vector of «fixed» parameters $c$ and an inversion with respect to the remaining free parameters $m$ is performed for each set of trial values. Then the set of trial values that produces the inversion with the smallest cost function is chosen. Even if not inverted simultaneously as the free parameters $m$ are, the parameters $c$ retrieved in this way can be considered as «inferred» by trial and error. Similar studies are performed for event 3 by considering the Sellano interferogram (Lundgren and Stramondo, 2002; Santini et al., 2004) or the Colfiorito-Sellano interferogram (Lundgren and Stramondo, 2002; Hernandez et al., 2004). Belardinelli et al. (2003) invert the fault mechanism, the location of the fault with respect to the observed hypocenter, the depth of the fault and a simplified slip distribution (fig. 4a), largely inspired to findings of Hunstad et al. (1999), using both GPS data and the Colfiorito interferogram.

Both GPS data, the Colfiorito interferogram and the Colfiorito-Sellano interferogram concern the cumulative coseismic displacement of events 1 and 2 , which occurred very close to each other in space and time and have a similar focal mechanism, according to the CMT solu-
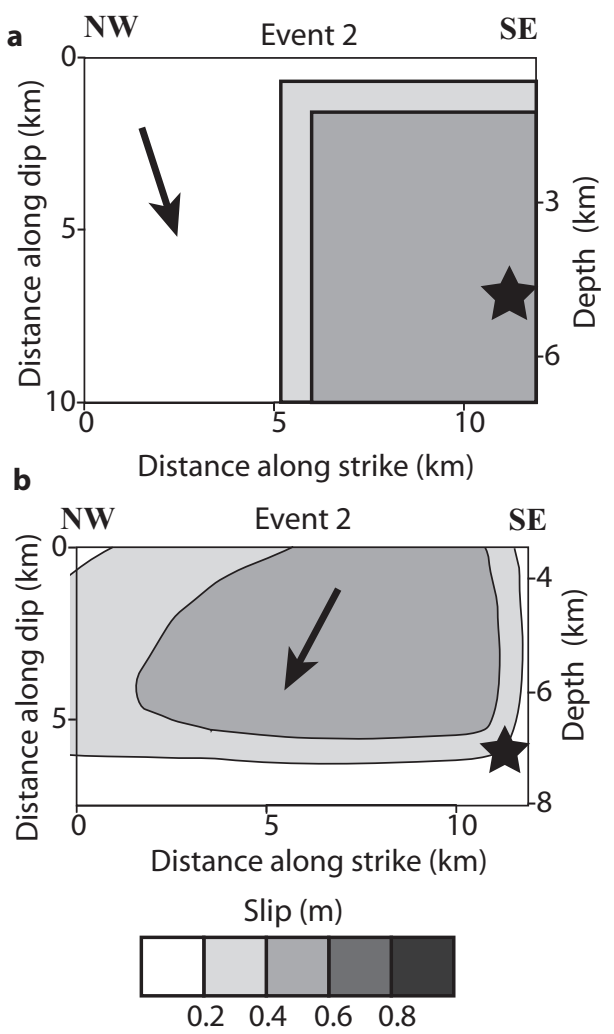

Fig. 4a,b. Simplified slip distribution of event 2 obtained by $a$ ) joint inversion of DInSAR and GPS data (Belardinelli et al., 2003, model $a$ in their table II) and b) inversion of strong-motion data (Capuano et al., 2000). An elastic homogenous half-space is assumed for inversion of geodetic data and a layered half-space (table II) for strong motion inversion. In each panel the black star represents the location of the hypocenter on the fault plane and the arrow represents the inverted direction of slip, assumed as homogeneous on the whole fault plane. In panel $a$ slip is obtained by linear interpolation of the inverted solution outside the patch with the largest slip $(53 \mathrm{~cm})$. Figure in panel $b$ is redrawn after Emolo and Zollo (2001).

tions. For this reason some UMI studies dealing with geodetic data assume that events 1 and 2 occurred on the same fault plane (e.g. fig. 3, Lundgren and Stramondo, 2002, Santini et al., 2004). This hypothesis was recently confirmed by high-resolution studies assessing the conti- 
nuity of the slip distribution across the two almost parallel faults of event 1 and 2 (Dalla Via et al. 2007). At first, UMI studies using the Colfiorito interferograms assumed it to consist of nine fringes of equal slant range displacement. The latter are discretized into 258 sites of known slant range displacement (fig. 5) in Belardinelli et al. (2003). Similarly the ColfioritoSellano and Sellano interferograms are assumed to consist of a discrete number of fringes of equal slant range displacement, subsequently digitized in a discrete number of data points (e.g. Lundgren and Stramondo, 2002). Later, from the Colfiorito interferogram Crippa et al. (2006) estimated slant-range displacement values in a 2-D grid containing more than 8900 data points. This denser data set allows a greater resolution in the inverted slip distribution compared to previous studies, but it also requires smoothing algorithms in order to reduce the roughness (the average gradient of the slip over the rupture area) of the inferred slip distribution (Dalla Via et al., 2007).

In order to limit the parameter space explored during the inversion, all the theoretically-based a priori constraints on $m$ should be used, mainly in cases of nonlinear inversions. For instance, while inverting for the source locations, the fault planes should be forced to pass through the instrumental hypocenters or to intersect the volume spanned by their hypocenter, taking into account the uncertainties in its location. In order to determine the inverted model of event 2, Belardinelli et al. (2003) consider the large error in its hypocentral depth (Amato et al., 1998) and vary the fault location in such a way that the vertical projection of the observed epicentre on the fault plane is located within the depth interval of uncertainty of the observed hypocenter, [3.7 km, $7.7 \mathrm{~km}$ ), (fig. $4 a)$. The same is true for the inverted model obtained by Lundgren and Stramondo (2002) for event 2 (fig. 3, top), but not for events 1 and 3 . As we can note in fig. 3 , for these events the vertical projections of the epicentres on the inverted fault planes locate at depths that are smaller than the minimum estimates of hypocentral depth provided by seismology ( 5 $\mathrm{km}$ for event 1 and $6.2 \mathrm{~km}$ for event 3, Amato et al., 1998).

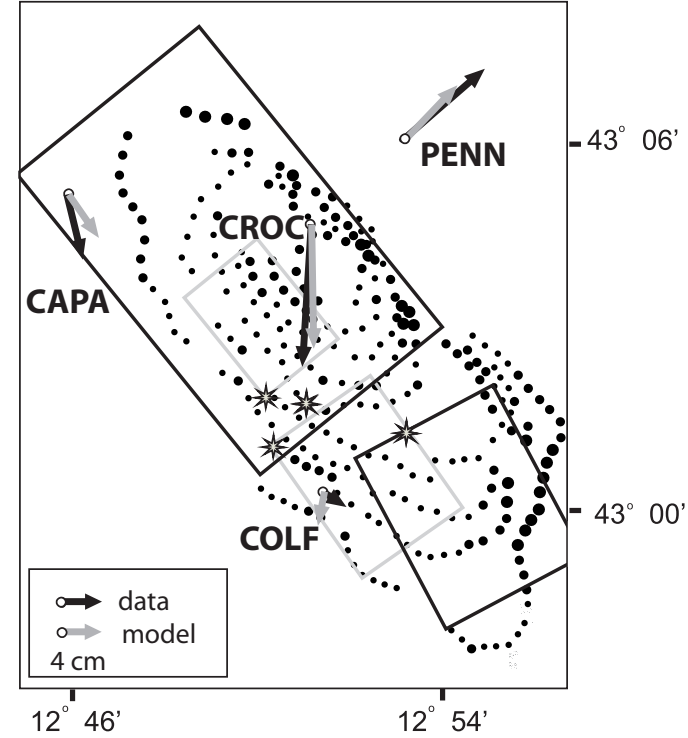

Fig. 5. Geometry used by Belardinelli et al. (2003) for the sources of events 1 and 2 and the other two minor events occurred on 3 and 6 October 1997 (grey boxes), whose epicentres are represented by black open stars. GPS data of horizontal displacement in the four stations located within $15 \mathrm{~km}$ from the mainshock epicentre are compared to model results. Results of the best model for event 2 according to joint inversion of GPS (at the four stations indicated) and DInSAR data are shown (model $a$ in table II of the mentioned paper). Slant range displacement seen by DInSAR data is assumed to be known in 258 «data points» (black dots). Dot size scales with the logarithm of the squared residual between model and DInSAR datum, as evaluated in the dot location. For the fault of event 2 , the inverted value of the top edge depth is $70 \mathrm{~m}$. Faults are assumed as rectangular dislocations in an elastic homogeneous half-space.

In order to perform an inversion it is necessary to refer to a particular crustal model. If the inverted parameters include the slip distribution over the fault plane, then the scalar seismic moment can be derived from the inverted solution, $m_{l}$, and the crustal model used. Thus in this case, the scalar seismic moment cannot be considered an additional free parameter to be determined by inversion, unlike the approach followed by Lundgren and Stramondo (2002). To this aim, 
both Belardinelli et al. (2003) for event 2 and Santini et al. (2003) for events 1-3 determine by inversion of geodetic data a slip distribution fulfilling the a priori constraint of a scalar seismic moment equal to the CMT solution. Similarly, for event 2, De Martini et al. (2003) inverted levelling data by grid search, assuming the scalar seismic moment of the CMT solution as a constraint to determine the fault length, width, top depth and a slip distribution homogeneous along dip, largely inspired to Pino and Mazza (2000) results (see next subsection).

\subsubsection{Inversion of seismic data}

For events 1-3, Pino and Mazza (2000) obtain the apparent source time function (STF) from waveforms recorded at three broadband stations (see section 2), using the method of the empirical Green functions (EGF) by deconvolution in the frequency domain. For each of the three major events, the authors select a suitable EGF (as a smaller event with a similar focal mechanism located close to the studied event). The STF is low-pass filtered below $1 \mathrm{~Hz}$ in order to remove small-scale source complexities of the EGF. The STF is also averaged along the three coordinate axes for each station and each event considered. For each event, the maximum amplitude and the duration of the obtained STF at the three stations are the data $d$ that can be compared with model results. The latter are developed using the Haskell source model and approximating the STF with a triangle having a width equal to the observed duration. The model estimates of the STF amplitude and duration $\left(d^{m o d}\right)$ depend on the following source parameters $(m)$ : the fault length, the horizontal component of the rupture velocity vector (assumed as homogeneous and unilateral) and the LP moment ratio (the ratio between the scalar seismic moment of the event studied and that of the EGF) for a fixed value of the average wave velocity $(c)$. The free parameters, $m$, are inverted by trial and error. Assuming fixed values of crustal rigidity and fault width, it is also possible to determine the maximum slip on the fault and its position along strike as a part of the solution. For event 2, a bimodal STF is suggested by AQU data, yielding a slip distribution with two maxima along strike.

Capuano et al. (2000) perform a trial and error inversion of S-waveforms recorded by the strong-motion accelerometers to infer the source geometry (focal mechanism, length and width), the location of the hypocenters on the fault plane, the rupture velocity vector (assumed as homogeneous), and a simplified slip distribution for events 1-3. Unlike the other events, for event 2 an inhomogeneous distribution of slip is required by data, with a gaussianlike peak located at about 3-4 km up-dip and NW of the nucleation point (fig. 3b). Only the acceleration field associated with the direct Swave motion is computed, assuming that it largely dominates in amplitude with respect to direct P-wave and converted/reflected waves in the near-source distance. The authors filter the signals of accelerometers in the range $1-5 \mathrm{~Hz}$ and integrate them to obtain ground velocities. The low frequency limit is imposed by the assumptions made by the authors in modelling the seismic radiation using the asymptotic theory (Frauhnofer approximation: wavelengths much smaller than distance from the source, comparable with the fault dimension in near field). The parameters of the starting model are inferred from previous analyses of the same authors. The latter estimate the STF and isochrones of events 1-3 at the considered seismic stations (see section 2) and study the Swave polarization of measured waveforms.

Hernandez et al. (2004) instead low pass the frequency content of accelerometer signals (assuming a 0.1-1.5 Hz filter) and integrate them twice (to obtain ground displacement), owing to difficulties in modelling the high frequency content of waveforms. The obtained data set is used to invert the slip distributions and the rupture histories of events 2 and 3 by means of non linear iterative inversion in the frequency domain, where each step is linearized. In this case, data, $d$, are represented by the waveform spectra for each frequency and component of displacement at each station. Free parameters, $m$, are the rupture time and slip distributions over the fault plane, assuming fixed the fault geometry and a homogeneous source time function with a rise time equal to $1 \mathrm{~s}$. 


\section{Results}

Inversion studies using only one kind of data or a part of the available data set should in principle check the consistency of their results with the observational evidences not directly taken into account during the inversion. To this aim, several UMI studies verify if the «misfit a posteriori» with observations not used for the inversion is small, where the misfit a posteriori is obtained by means of a forward model using the inverted source parameters $m_{I}$. For instance, this is the case of Santini et al. (2004) who compare inversion results obtained for events 1 and 2 with vertical GPS data and with the Colfiorito-Sellano interferogram (not used in the inversion). For events 13, Hernandez et al. (2004) check the inversion results obtained from geodetic data with strong-motion data, assuming the inverted slip model, a homogeneous rupture velocity and $1 \mathrm{~s}$ rise time. Capuano et al. (2000) check their inverted final model for events 1 and 2 comparing the waveforms obtained by forward modelling at the station of Nocera Umbra (not used in their inversion) with recorded data at the same station.

Moreover, UMI studies not using the a priori constraint of the total scalar seismic moment (see Section 3.2.1.) verify a posteriori the consistency of their estimate of the seismic moment with the CMT solution.

The estimates of the inverted parameters, $m_{I}$, are affected by uncertainties that can be related to errors in data or parameters fixed in the inversion. Uncertainty is also intrinsic to the inversion method, taking into account the limitations concerning the resolving power of the data used and the modelling appropriateness. Capuano et al. (2000) and Pino and Mazza (2000) estimate an uncertainty of $10-20 \%$ in each component of $m_{I}$ by determining the maximum and minimum values of each of the free parameters producing model results $d^{\text {mod }}$ compatible with the reading errors of data. For event 2, Belardinelli et al. (2003) define as «acceptable» a model $m$ that reproduces on average the data within the measurement error (having $\varepsilon<\varepsilon_{\max }$ ). An ensemble of acceptable models, $m_{a}^{j}, j=1,2, \ldots, n_{\mathrm{A}}$, is built by select- ing a subset of the inverted models $m_{I}^{i}$ generated by varying randomly the starting models $\mathbf{m}_{s}^{i}, i=1,2, \ldots, 500$. The authors provide estimates of the uncertainty of each free parameter by analyzing the distribution of that parameter within the ensemble of acceptable models. Some UMI studies demonstrate that the resolution of the inversion of geodetic data decreases with increasing the source depth. In particular in the deepest parts of the seismic sources of events 1 and 2 (depth> $3 \mathrm{~km})$, the resolution gets significantly worse. For these events, both the uncertainty of each inverted parameter (Hernandez et al., 2004) and the «minimum resolvable area of the fault» (with respect to the slip distribution, Crippa et al., 2006) increase significantly at depths larger than $3 \mathrm{~km}$. The minimum resolvable area of the fault is defined as the area of a fault patch producing on surface measure points an average displacement of the order of data uncertainty, for $1 \mathrm{~m}$ of homogeneous slip.

The following state of knowledge of the three seismic sources of events 1-3 can be outlined on the basis of UMI studies. All three sources are recognized as NW-SE trending and SW dipping (e.g. fig. 2). However the values fixed, or inferred (in the sense explained in section 3.2.1.), or inverted for the strike of event 3 belong to a quite large interval $123^{\circ}$ $160^{\circ}$. The hypocenter is located near the border of the faults of event 2 (SE border, e.g. figs. 2, 4 and 5) and event 3 (NW border, e.g. figs. 3 and 6). The rupture of events 2 and 3 propagated from the hypocenters with an average rupture velocity of about $2.6-3 \mathrm{~km} / \mathrm{s}(\mathrm{Ca}-$ puano et al., 2000; Pino and Mazza, 2000). In most of the studies, the hypocenter of Event 1 is assumed to be located near the NW border of the fault, suggesting a unilateral rupture (e.g. figs. 2 and 5), however Capuano et al., 2000 attribute a bilateral rupture mechanism to event 1 .

The most studied source is that of event 2, characterized by a fault length of about 12-15 km (e.g. Capuano et al., 2000; Pino and Mazza, 2000, see e.g. fig. 4). For event 2, GPS data, in particular the large horizontal displacement recorded at the station PENN (Hunstad et al., 1999), require the top of the fault to be 

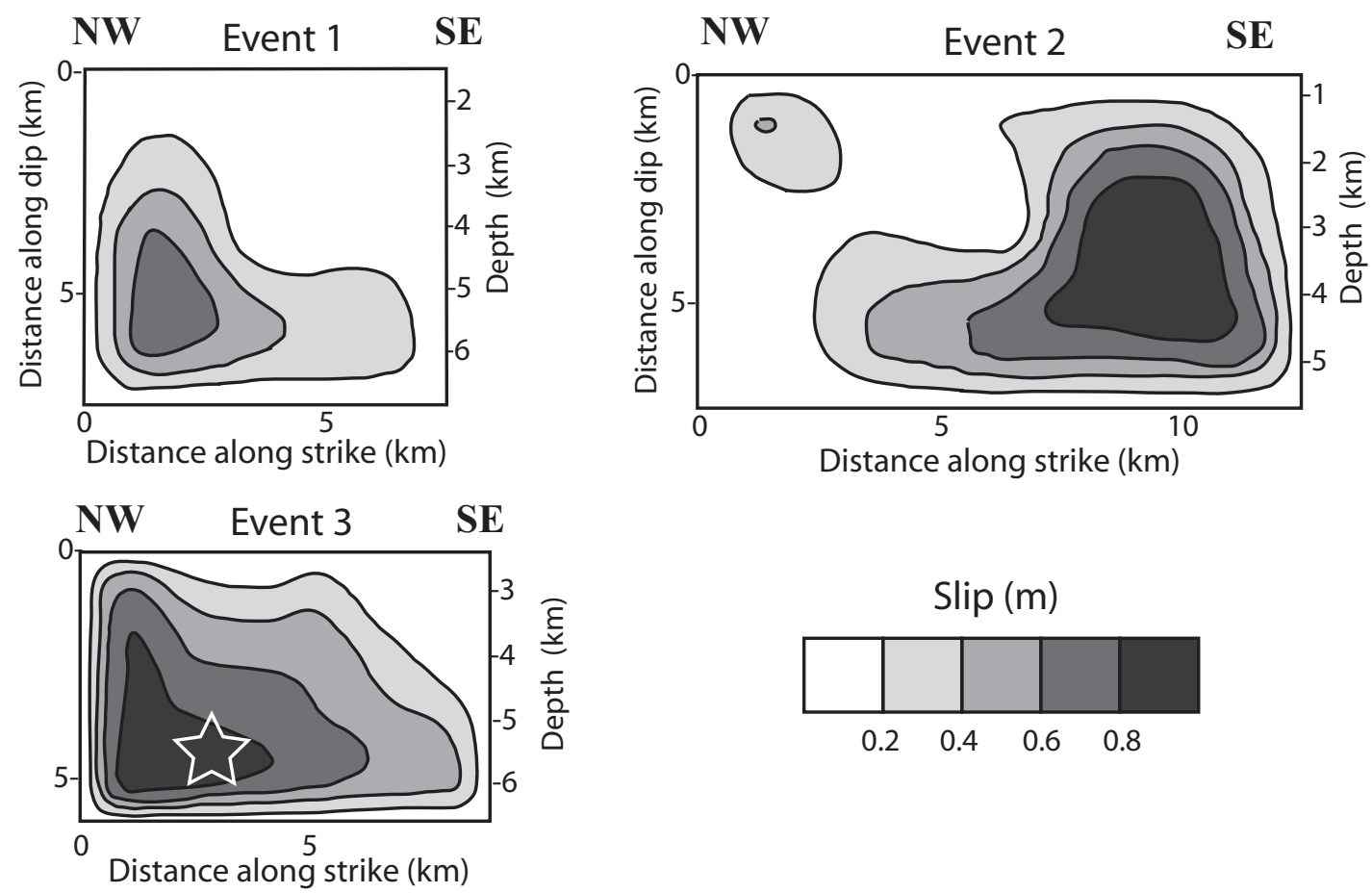

Fig. 6. Best fit model for slip distribution of events 1-3 from inversion of geodetic data (Hernandez et al., 2004). A joint inversion of DInSAR and GPS data is performed in case of events 1 and 2 (see fig. 3), while event 3 rupture is inverted from DInSAR data alone. The open white star indicates the hypocenter on the fault plane of event 3. The crustal model used is reported in table I. Figure redrawn after Hernandez et al. (2004).

shallower $(0.04-0.7 \mathrm{~km})$ than using levelling data $(3.4 \mathrm{~km}$, De Martini et al., 2003), SAR (1-2 km) or strong motion data (0.7-3.38 km). Except for the Capuano et al. (2000) solution (fig. 4b), the inverted value of the rake angle of event 2 entails a small left lateral component superimposed to the dominant normal component (figs. 3 and 4a) in agreement with the CMT solution. Even if less resolved by GPS and DInSAR data, the inferred value of the dip angle of event 2 is around 40 degrees (Belardinelli et al., 2003; Hernandez et al., 2004, De Martini et al. 2003; Capuano et al., 2000) in agreement with the CMT solution, showing an anomalously small value for a normal fault.

On the average, the top of the fault of event 2 is found to be shallower than that of events 1 and 3 (e.g. fig. 6). The inverted slip distribu- tions are heterogeneous and characterized by patches containing the maximum values near the hypocenter locations (figs. 3, 4 and 6). For event 2 , this patch has about $5 \mathrm{~km}$ extent along strike and concentrates below about $2 \mathrm{~km}$ depth (Lundgren and Stramondo, 2002, Belardinelli et al., 2003, Santini et al.; 2003, Hernandez et al. 2004; Crippa et al., 2006). For event 2, another smaller patch of slip located close to NW end of the fault can be inferred on the basis of some of the inversion studies (Pino et al., 1999; Lundgren and Stramondo, 2002; Hernandez et al., 2004; De Martini et al., 2003; Crippa et al., 2006; Dalla Via et al., 2007, see figs. 3 and 6). Outside and around the two high-slip patches, the distribution of aftershocks recorded in the first three months after event 2 shows a larger density (De Martini et al., 2004) suggesting the presence of an asperity. 


\section{Discussion and conclusive remarks}

This paper reviews several studies of the extended sources of the three major events of the Umbria-Marche sequence that are performed through inversion of observed coseismic displacements (UMI studies). Comparing the methods and the results obtained by each UMI study we see that: 1) the best resolved source is that of the largest event (event 2: September 26, 1997 at 09:40 GMT, $\mathrm{M}_{\mathrm{W}}=6.0$ ); 2) only some studies estimate the uncertainties of the inverted parameters and these studies are not necessarily the most recent ones; 3) in general, only one kind of data set is inverted and joint inversions of different data sets are performed only using different kinds of geodetic data (GPS and DInSAR). If point 1 ) is quite obvious given the moderate size of seismicity recorded during the 1997 the Umbria-Marche sequence, points 2 and 3 are worth discussing in the following part of this section, guided by other inversion studies of extended sources of recent earthquakes occurred in the world.

Piatanesi et al. (2007) point out that most of computational effort in finite-fault inversions seems to be devoted to finding the model which yields the minimum cost function and that only few studies deal with a posterior error analysis, while the latter may have important implications, for instance, for providing ground motion scenarios. Then they propose a technique to estimate the variability of rupture models that are consistent with data. Referring the results to each of the inferred parameters, the authors consider well resolved a parameter in a certain point of the fault if the ratio between its variability range and the value of the inverted parameter is low $(\leq 0.5)$. The regions of the fault where this occurs are stably inverted with respect to that parameter. For the Umbria-Marche sequence a similar analysis was made only for events 1 and 2 by Hernandez et al. (2004) when they perform a joint inversion of DInSAR and GPS data (see sections 2 and 4). However, synthetic tests shown by Piatanesi et al. (2007) point out that the variability of one parameter can correctly estimate the error bar of the same parameter only if the modelling is consistent and in particular if a realistic crustal model is used to evaluate the field of coseismic displacement. This is due to the well known fact that uncertainties in the crustal model represent a main source of noise in inversion studies of extended seismic sources (e.g. Wald and Graves, 2001).

Generally, UMI studies assume the seismic sources to be described by dislocations or point sources in an elastic homogeneous half-space, except for studies considering strong-motion data (e.g. Capuano et al., 2000 and Hernandez et al., 2004), where a layered half-space is considered with rigidity values smaller than 30 $\mathrm{GPa}$ (average value for the crust) in the first 7$8 \mathrm{~km}$ depth (see tables I and II). In general, if the layering of the crust is taken into account, the top edges of the three faults turn out to be deeper (1.5-3.5 km, 0.7-3.4 km, 2.4-4.8 km for events 1,2 and 3 respectively) than the values obtained using half-space models $(0.04-2 \mathrm{~km}$, 0.04-1 km, 0.64-1.15 km, for events 1,2 and 3 respectively). Alternatively, since in UMI studies different data sets are considered, one could explain the different estimates of the top edge depth referring to the different «importance» (e.g. Tarantola, 1987) of a particular data set in allowing the resolution of slip at depth. However synthetic tests inverting geodetic data and strong motion data separately (Delouis et al., 2002) do not seem to show that strong motion data are more important or more sensitive to final slip at depth than geodetic data are.

Neglecting low rigidity layers near the surface leads to underestimate mainly the horizontal components of displacement at the surface. This is confirmed by several studies (e.g. Wald and Grave 2001; Megna et al., 2008). In the framework of inversion studies, for the same displacement observed at the surface, overestimating the rigidity of near surface layers leads to slip overestimates near the surface (e.g. Wald and Graves, 2001; Simons et al., 2002; Piatanesi et al., 2007) or to underestimate the top depth of the dislocation, in agreement with theoretical modelling of dislocations in layered media (Savage, 1998). However Piatanesi et al. (2007) show that the along strike slip distribution can be affected by uncertainties in the crustal model too. For event 2, we can note that the amplitudes of horizontal displacement at GPS sites are compa- 
Table I. Crustal model used by Hernandez et al. (2004). The rigidity modulus is indicated with $\mu$.

\begin{tabular}{ccccc}
\hline \hline Top depth $(\mathrm{m})$ & $\mathrm{V}_{\mathrm{p}}(\mathrm{m} / \mathrm{s})$ & $\mathrm{Vs}_{(\mathrm{m} / \mathrm{s})}$ & $\rho\left(\mathrm{kg} / \mathrm{m}^{3}\right)$ & $\mu(\mathrm{GPa})$ \\
\hline 0 & 4800 & 2666 & 2600 & 18.5 \\
4000 & 5500 & 3055 & 2800 & 26.1 \\
7000 & 6300 & 3500 & 2900 & 35.5 \\
30000 & 8000 & 4444 & 3100 & 61.2 \\
\hline
\end{tabular}

Table II. Crustal model reported by Emolo and Zollo (2001) and used by Capuano et al. (2000). The rigidity modulus is indicated with $\mu$.

\begin{tabular}{ccccc}
\hline \hline Top depth $(\mathrm{m})$ & $\mathrm{V}_{\mathrm{p}}(\mathrm{m} / \mathrm{s})$ & $\mathrm{V}_{\mathrm{s}}(\mathrm{m} / \mathrm{s})$ & $\rho\left(\mathrm{kg} / \mathrm{m}^{3}\right)$ & $\mu(\mathrm{GPa})$ \\
\hline 0 & 2000 & 1100 & 2000 & 2.4 \\
200 & 4400 & 2400 & 2400 & 13.8 \\
1800 & 5900 & 3200 & 2400 & 24.6 \\
8000 & 6250 & 3500 & 2500 & 30.6 \\
\hline
\end{tabular}

rable if either a 1-D crustal model (fig. 2) or a half-space model (fig. 5) is used, despite of the fact that in fig. 2 the fault top is much deeper than in fig. 5. In both figures the amplitudes of horizontal displacement are underestimated with respect to data. This is particularly true for the station of PENN where secondary effects such as deep-seated gravitational slope deformations are likely to have amplified the observed horizontal displacement with respect to estimates of coseismic displacement due to dislocations in elastic media (Moro et al., 2007). In principle these effects should be taken into account before inverting geodetic data using elasto-static dislocation models. Neglecting these secondary effects at PENN might lead to underestimate the fault top depth of event 2 in UMI studies that invert geodetic data alone.

The joint inversion of different kinds of data increases in a robust way both the information carried by the inverted model (because different data can have different importance for each model parameter) and its resolving power (because the number of data of comparable importance to estimate the same parameter increases). Usually, the single data set is reproduced by the model resulting from a joint inversion worse than it is when that kind of data is inverted separately, however the stability of the joint inversion is generally greater. For instance, joint inversions of GPS data and DInSAR data allow to estimate horizontal components of the slip vector better than using DInSAR data alone (e.g. Lundgren and Stramondo, 2002; Belardinelli et al., 2003) since GPS data are most precise in horizontal deformations (Pedersen et al., 2003). On the other hand, slip inversions using seismological data only are likely to be affected by trade off between rupture times and slip location (Delouis et al., 2002).

To conclude, the lesson learnt from the analysis of UMI studies suggests that separate inversions of geodetic and seismological data can lead to fault models that are not strictly comparable, also due to the different crustal models that are used in evaluating static and dynamic displacement values. Finite-fault studies dealing with recent earthquakes that occurred in the world are in favour of joint inversions assuming at least 1-D Earth structures (Wald and Graves, 2001). The uncertainties related to the choice of 
the crust structure could be faced by comparing the results of inversions obtained with all the crustal models available for a given region.

\section{Acknowledgements}

I want to thank Laura Sandri and Paolo Baldi for introducing me to the subject of joint inversion of geodetic data and the anonymous reviewers for constructive comments. I am also grateful to Antonella Megna for having provided material in advance of publication and Salvatore Stramondo and Paul Lundgren for having provided slip models according to their joint inversion of DInSAR and GPS data.

\section{REFERENCES}

Amato, A., R. Azzara, C. Chiarabba, G. B. Cimini, M. Cocco, M. Di Bona, L. Margheriti, S. Mazza, F. Mele, G. Selvaggi, A. Basili, E. Boschi, F. Courboulex, A. Deschamps, S. Gaffet, G. Bittarelli, L. Chiaraluce, D. Piccinini and M. Ripepe (1998): The 1997 Umbria-Marche, Italy, earthquake sequence: a first look at the main shocks and aftershocks, Geophys. Res. Lett., 25 (15), 2861-2864.

Belardinelli, M. E., L. SAndri and P. Baldi (2003): The major event of the 1997 Umbria-Marche (Italy) sequence; what could we learn from DInSAR and GPS data?, Geophys. J. Int., 153, 1, 242-252.

Capuano, P., A. Zollo, A. Emolo, S. Marcucci and G. Milana (2000): Rupture mechanism and source parameters of Umbria-Marche mainshocks from strong motion data, J. Seismol., 4, 463-478.

Crippa, B., M. Crosetto, E. Biescas, C. Troise, F. Pingue and G. DE NATAle (2006): An advanced slip model for the Umbria-Marche earthquake sequence: coseismic displacements observed by SAR interferometry and model inversion, Geophys. J. Int., 164 (1), 36-45.

Dalla Via, G., B. Crippa, E. M. Toraldo Serra, G. GiacoMUZZI and R. SABADINI (2007): Exploitation of high-density DInSAR data points of the Umbria-Marche (Italy) 1997 seismic sequence for fault characteristics, Geophys. Res. Lett., 34, L17301, doi: 10.1029/2007GL030718.

Delouis, B., D. Giardini, P. Lundgren and J. Salichon (2002): Joint inversion of Insar, GPS, teleseismic and strong-motion data for the spatial and temporal distribution of earthquake slip: application to the 1999 Izmit mainshock, Bull. Seismol. Soc. Am., 92 (1), 278-299.

De Martini, P. M., N. A. Pino, G. Valensise and S. MazZA (2003): Geodetic and seismologic evidence for slip variability along a blind normal fault in the UmbriaMarche 1997-1998 earthquakes (central Italy), Geophys. J. Int., 155, 819-829.

Emolo, A. and A. Zollo (2001): Accelerometric radiation simulation for the September 26, 1997 Umbria-Marche
(Central Italy) main shocks, Annals Geophys., 44 (3), 605-617.

Ekström, G., A. Morelli, E. Boschi and A. M. DziewonSKI (1998): Moment tensor analysis of the UmbriaMarche earthquake sequence of September-October 1997, Geophys. Res. Lett., 25, 11, 1971-1974.

Hernandez, B., M. Cocco, F. Cotton, S. Stramondo, O. Scotti, F. Courboulex and M. CAmpillo (2004): Rupture history of the 1997 Umbria-Marche (central Italy) main shocks from the inversion of GPS, DInSAR and near field strong motion data, Annals Geophys., 47 (4), 1355-1376.

Hunstad, I., M. Anzidei, M. Cocco, P. Baldi, A. Galvani and A. PESCI (1999): Modelling coseismic displacements during the 1997 Umbria-Marche earthquake (central Italy), Geophys. J. Int., 139, 2, 283-295

LundGREN, P. and S. STRAMONDO (2002): Slip distribution of the 1997 Umbria-Marche earthquake sequence: Joint inversion of GPS and synthetic aperture radar interferometry data, J. Geophys. Res., 107 (B11), 2316, doi:10.1029/2000JB000103.

Megna, A., S. Barba, S. SAntini and M. Dragoni (2008): Effect of geological complexities on coseismic displacement: hints from 2D modelling, Terra Nova, 20 (3) , 173-179, doi:10.1111/j.1365-3121.2008.00800.x.

Moro, M., M. Saroli, S. Salvi, S. Stramondo and F. Doumaz (2007): The relationship between seismic deformation and deep-seated gravitational movements during the Umbria-Marche (central Italy) earthquakes, Geomorphology, 89, 297-307.

Pedersen, R., S. Jónsson, T. Árnadóttir, F. Freysteinn and K. FEIGL (2003): Fault slip distribution of two June 2000 MW6.5 earthquakes in South Iceland estimated from joint inversion of InSAR and GPS measurements, Earth Planet. Sci. Lett., 213, 487-502.

Piatanesi, A., A. Cirella, P. Spudich and M. Cocco (2007): A global search inversion for earthquake kinematic rupture history: Application to the 2000 western Tottori, Japan earthquake, J. Geophys. Res., 112, B07314, doi:10.1029/2006JB004821.

PINO, N.A. and S. MAZZA (2000): The Umbria-Marche (central Italy) earthquakes: relation between rupture directivity and sequence evolution for the $\mathrm{Mw}>5$ shocks, J. Seismol., 4, 451-461.

Santini, S., P. Baldi, M. Dragoni, A. Piombo, S. Salvi, G. Spada and S. Stramondo (2004): Monte Carlo Inversion of DInSAR Data for Dislocation Modeling: Application to the 1997 Umbria-Marche Seismic Sequence (Central Italy), Pure Appl. Geophys., 161, 817-838.

SAvage, J.C. (1998): Displacement field for an edge dislocation in a layered half-space, J. Geophys. Res., 103 (B2), 2439-2446

Simons, M., Y. FialKo and L. Rivera (2002): Co-seismic deformation from the 1999 MW 7.1 Hector Mine, California, earthquake as inferred from InSAR and GPS observations, Bull. Seism. Soc. Am., 92, 1390-1402.

TARAntola, A. (1987): Inverse problem theory: Methods for data fitting and model parameter estimation (Elsevier), pp. 63-76.

WALD, D. J. and R. W. GRAVES (2001): Resolution analysis of finite source inversion using one- and three-dimensional Green's functions: 2. Combining seismic and geodetic data, J. Geophys. Res., 106 (B5), 8767-8788. 